\title{
ON HOLOMORPHIC REPRESENTATIONS OF SYMPLECTIC GROUPS
}

\author{
BY TUONG TON-THAT ${ }^{1}$
}

Communicated by Shlomo Sternberg, April 30, $1975^{2}$

Let $G$ denote the complex symplectic group which may be defined by the equation

$$
G=\left\{g \in \mathrm{GL}(2 k, \mathrm{C}): g s_{k} g^{t}=s_{k}, s_{k}=\left[\begin{array}{cc}
0 & -I_{k} \\
I_{k} & 0
\end{array}\right]\right\}
$$

In this paper we shall give a simple and concrete realization of a set of representatives of all irreducible holomorphic representations of $G$. This realization, which involves the $G$-module structure of a symmetric algebra of polynomial functions is inspired by the work of B. Kostant [1] and follows the general scheme formulated in [2]. Detailed proofs will appear elsewhere.

1. The symmetric algebra $S\left(E^{*}\right)$. Set $E=\mathbf{C}^{n \times 2 k}$ with $k \geqslant n \geqslant 2$; then $G$ acts linearly on $E$ by right multiplication. Let $(\cdot, \cdot)$ denote the skew-symmetric bilinear form on $E$ given by

$$
(X, Y)=\operatorname{trace}\left(X s_{k} Y^{t}\right), \quad \forall X, Y \in E .
$$

If $X \in E$, let $X^{*}$ denote the linear form $Y \rightarrow(X, Y)$ on $E$. The map $X \rightarrow X^{*}$ establishes an isomorphism between $E$ and its dual $E^{*}$. Let $S\left(E^{*}\right)$ denote the symmetric algebra of all complex-valued polynomial functions on $E$. The action of $G$ on $E$ induces a representation $R$ of $G$ on $S\left(E^{*}\right)$ defined by

$$
(R(g) p)(X)=p(X g), \quad \forall p \in S\left(E^{*}\right), \quad \forall X \in E .
$$

If $X \in E$, define a differential operator $X^{*}(D)$ on $S\left(E^{*}\right)$ by setting

$$
\left(X^{*}(D) f\right)(Y)=\{(d / d t) f(Y+t X)\}_{t=0},
$$

$$
\text { for all } f \in S\left(E^{*}\right), t \in \mathbf{R} \text {, and } X, Y \in E \text {. }
$$

Define $\left(X_{1}^{*} \cdots X_{n}^{*}\right)(D) f=X_{1}^{*}(D)\left(\left(X_{2}^{*} \cdots X_{n}^{*}\right)(D) f\right)$ inductively on $n$. If

AMS (MOS) subject classifications (1970). Primary 22E45; Secondary 13 F20.

Key words and phrases. Symmetric algebras of polynomials, irreducible holomorphic representations of symplectic groups.

1 The author is a Postdoctoral Research Fellow at Harvard University.

2 Originally received February 2, 1975. 
$m$ and $l$ are nonnegative integers and if $S_{m}$ denotes the symmetric group on $m$ elements, then it may be shown that

$$
\begin{aligned}
& {\left[X_{1}^{*} \cdots X_{l}^{*}(D)\right] Y_{1}^{*} \cdots Y_{m}^{*}} \\
& \quad=\left\{\begin{array}{l}
0, \text { if } m<l, \\
\frac{(-1)^{l}}{(m-l) !} \sum_{\sigma \in S_{m} X_{1}^{*}\left(Y_{\sigma(1)}\right) \cdots X_{l}^{*}\left(Y_{\sigma(l)}^{*}\right) Y_{\sigma(l+1)}^{*} \cdots Y_{\sigma(m)}^{*},}^{\text {if } m \geqslant l}
\end{array}\right.
\end{aligned}
$$

It follows from the above equation and by linearity that the map $X^{*} \rightarrow X^{*}(D)$ extends to an isomorphism $p \rightarrow p(D)$ between $S\left(E^{*}\right)$ and the symmetric algebra $S(E)$ of differential operators on $E$.

A polynomial $f \in S\left(E^{*}\right)$ will be called $G$-invariant if $R(g) f=f, \forall g \in G$. A differential operator $p(D) \in S(E)$ will be called $G$-invariant if $R(g)(p(D) f)=$ $p(D)(R(g) f)$, for all $g \in G, f \in S\left(E^{*}\right)$. It is then shown that $p \in S\left(E^{*}\right)$ is $G$-invariant if and only if $p(D)$ is $G$-invariant.

Let $J\left(E^{*}\right)$ (resp. $J(E)$ ) denote the subalgebra of $S\left(E^{*}\right)$ (resp. of $S(E)$ ) consisting of all $G$-invariant polynomials (resp. of all $G$-invariant differential operators). Let $J^{+}\left(E^{*}\right)$ denote the set of all $G$-invariant polynomials without constant terms; $J^{+}(E)$ is then defined in a similar fashion.

A polynomial $f \in S\left(E^{*}\right)$ is said to be G-harmonic if $p(D) f=0$ for all $p \in$ $J^{+}\left(E^{*}\right)$. Let $H\left(E^{*}\right)$ denote the subspace of $S\left(E^{*}\right)$ consisting of all $G$-harmonic polynomials. Let $J^{+}\left(E^{*}\right) S\left(E^{*}\right)$ be the ideal in $S\left(E^{*}\right)$ generated by $J^{+}\left(E^{*}\right)$, and denote by $V$ the algebraic variety in $E$ of common zeros of polynomials in the ideal $J^{+}\left(E^{*}\right) S\left(E^{*}\right)$. It follows from the theory of polynomial invariants (cf. [3, Chapter VI]) that $J\left(E^{*}\right)$ is generated by the constant function 1 and $n(n-1) / 2$ polynomials $p_{i j}$ defined by

$$
p_{i j}(X)=\sum_{l=1}^{k}\left(X_{i, l+k} X_{j l}-X_{i l} X_{j, l+k}\right), \quad 1 \leqslant i<j \leqslant n ; X=\left(X_{r s}\right) \in E .
$$

Moreover, we have $V=\left\{X \in E ; X s_{k} X^{t}=0\right\}$ and that $H\left(E^{*}\right)=\left\{f \in S\left(E^{*}\right): p_{i j}(D) f\right.$ $=0, \forall i, j, 1 \leqslant i<j \leqslant n\}$. It is then shown that the ideal $J^{+}\left(E^{*}\right) S\left(E^{*}\right)$ is prime.

THEOREM 1.1. The space $S\left(E^{*}\right)$ is decomposed into a direct sum as $S\left(E^{*}\right)$ $=J^{+}\left(E^{*}\right) S\left(E^{*}\right) \oplus H\left(E^{*}\right)$. Moreover, $S\left(E^{*}\right)=J\left(E^{*}\right) \otimes H\left(E^{*}\right)$ and $H\left(E^{*}\right)$ is spanned by all polynomials $\left(X^{*}\right)^{m}, m=1,2, \ldots$, for all $X \in V$.

CoROllaRY 1.2. If $S(V)$ denotes the ring of functions on $V$ obtained by restricting elements of $S\left(E^{*}\right)$ to $V$, then the restriction mapping $f \rightarrow f / V(f \in$ $\left.H\left(E^{*}\right)\right)$ is a G-module isomorphism of $H\left(E^{*}\right)$ onto $S(V)$.

2. The irreducible holomorphic representations of $G$. Let $B$ denote the lower triangular subgroup of $\operatorname{GL}(n, \mathbf{C})$ and define a holomorphic character $\xi=$ 
$\xi\left(m_{1}, \ldots, m_{n}\right)$ of $B$ by setting

$$
\xi(b)=b_{11}^{m_{1}} b_{22}^{m_{2}} \cdots b_{n n}^{m_{n}} \quad(b \in B),
$$

where the $m_{i}$ 's $(1 \leqslant i \leqslant n)$ are integers satisfying $m_{1} \geqslant m_{2} \geqslant \cdots \geqslant m_{n} \geqslant 0$. A polynomial $f \in S\left(E^{*}\right)$ will be called $\xi$-covariant if $f(b X)=\xi(b) f(X), \forall(b, X) \in$ $B \times E$. Let $H(E, \xi)$ denote the subspace of $H\left(E^{*}\right)$ consisting of all $\xi$-covariant $G$-harmonic polynomials.

THEOREM 2.1. If $R(\cdot, \xi)$ denotes the representation of $G$ which is obtained by right translation on $H(E, \xi)$ then $R(\cdot, \xi)$ is irreducible and its highest weight is indexed by $\left(m_{1}, m_{2}, \ldots, m_{n}, 0, \ldots, 0\right)(k$ factors).

Proof. Let

and

$$
C=\left\{\left[\begin{array}{cc}
c & 0 \\
0 & c^{-1}
\end{array}\right] \in \mathrm{GL}(2 k, \mathrm{C}): c \text { diagonal } k \times k \text { matrix }\right\}
$$

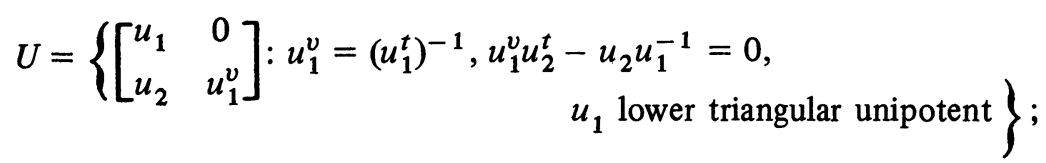

then $C U$ is a Borel subgroup of $G$. Define a holomorphic character $\zeta$ on $C U$ by setting

$$
\zeta(c u)=c_{11}^{m_{1}} \cdots c_{n n}^{m_{n}}, \quad \forall c u \in C U .
$$

Let $\operatorname{Hol}(G, \zeta)$ denote the space of all $\zeta$-covariant holomorphic functions on $G$. Then by the Borel-Weil-Bott theorem the representation $\pi(\cdot, \zeta)$ of $G$ which is obtained by right translation on $\operatorname{Hol}(G, \zeta)$ is irreducible (see also [4, Chapter $\mathrm{XVI}])$. Let $\mathbf{I}=\left[I_{n} 0\right] \in E$, then $\operatorname{Orb}(\mathbf{I})=\{\mathbf{I} g: g \in G\}$ is a dense subset of $V$. Define a map $\Phi$ from $H(E, \xi)$ into $\operatorname{Hol}(G, \zeta)$ by the equation $(\Phi f)(g)=f(\mathrm{I} g)$, $\forall f \in H(E, \xi), \forall g \in G$. Then it follows from Corollary 1.2 that $\Phi$ is a $G$-module isomorphism.

When $k=n$, the following theorem is an immediate consequence of Theorem 2.1 .

THEOREM 2.2. Suppose that

$$
E=\mathrm{C}^{k \times 2 k} \quad(k \geqslant 2) \text { and } \xi=\xi\left(m_{1}, m_{2}, \ldots, m_{k}\right)
$$

then the representations $R(\cdot, \xi)$ of $G$ on the various spaces $H(E, \xi)$ realize up to equivalence all irreducible holomorphic representations of $G$ when the $m_{i}$ 's $(1 \leqslant i \leqslant k)$ are allowed to take all integral values subject to the condition $m_{1} \geqslant m_{2} \geqslant \cdots \geqslant m_{k} \geqslant 0$. Moreover, to each representation $R(\cdot, \xi)$ corresponds a highest weight vector $f_{\xi} \in S\left(E^{*}\right)$ defined by the equation 


$$
f_{\xi}(X)=\Delta_{1}^{m_{1}-m_{2}}(X) \Delta_{2}^{m_{2}-m_{3}}(X) \cdots \Delta_{k-1}^{m_{k-1}-m_{k}}(X) \Delta_{k}^{m} k(X), \quad \forall X \in E
$$

where the $\Delta_{i}(X) \quad(1 \leqslant i \leqslant k)$ are the principal minors of $X$.

\section{REFERENCES}

1. B. Kostant, Lie group representations on polynomial rings, Amer. J. Math. 85 (1963), 327-404. MR 28 \#1252.

2. T. Ton-That, Lie group representations and harmonic polynomials of a matrix variable, Ph. D. Dissertation, Univ. of California, Irvine, Calif., 1974.

3. $\mathrm{H}$. Weyl, The classical groups. Their invariants and representations, Princeton Univ. Press, Princeton, N. J., 1939. MR 1, 42.

4. D. Želobenko, Compact Lie groups and their representations, "Nauka", Moscow, 1970; English transl., Transl. Math. Monographs, vol. 40, Amer. Math. Soc., Providence, R. I., 1973.

DEPARTMENT OF MATHEMATICS, HARVARD UNIVERSITY, CAMBRIDGE, MASSACHUSETTS 02138 